\title{
A diet low in fermentable sugars and polyols improves symptoms in diarrhoea predominant irritable bowel syndrome
}

\author{
A. C. O. Okonkwo ${ }^{1}$, B. Davidson ${ }^{2}$ and N. P. Thompson ${ }^{3}$ \\ ${ }^{1}$ Newcastle University Medical School, Newcastle, UK, NE2 4HH, ${ }^{2}$ Department of Dietetics, Newcastle Hospitals, UK, \\ NE4 5PL and ${ }^{3}$ Department of Gastroenterology, Newcastle Hospitals, UK, NE4 5PL
}

Irritable bowel syndrome (IBS) is a functional gastrointestinal disorder that has a prevalence of $10-15 \%$ in industrialised countries ${ }^{(1)}$. Diarrhoea predominant irritable bowel syndrome (IBS-D) primarily effects the working population (females $20-40$ years old) ${ }^{(1)}$. Fermentable oligosaccharides, monosaccharides and polyols (FODMAPs) cause bloating and loose stools through increased hydrogen gas and an osmotic gradient for increased water entry into the gut lumen ${ }^{(2)}$. A low FODMAP diet has been proven to reduce IBS-D symptoms; however, these reductions have not been quantified ${ }^{(2)}$.

Observe if the symptomatic benefit demonstrated in the literature could be reproduced in the North-East of England and to quantify the efficacy of a low FODMAP diet in IBS-D.

Symptom change according to the Birmingham IBS Symptom Questionnaire was recorded after 4 weeks ${ }^{(3)}$. Symptom severity was numerated for analysis: all of the time $=6$; most of the time $=5$; a good bit of the time $=4$; some of the time $=3$; a little of the time $=2$; none of the time $=1$. Statistical significance was calculated using Prism 6.0 (GRAPHPAD) by unpaired $t$-test.

Of 42 suitable patients 21 responded to the questionnaire, all were compliant with the diet for a minimum of 4 weeks. 10 reported complete resolution of symptoms. 15 patients saw an improvement in questionnaire symptom category in abdominal discomfort $(-1.8 \pm 0.5, p<0.01), 11$ whilst sleeping $(-0.9 \pm 0.4, p<0.05)$ and 14 after eating $(-1.4 \pm 0.6, p<0.05) .13$ experienced fewer loose bowel motions $(-1.4 \pm 0.5, p<0.05)$ and 13 experienced less urgency $(-1.2 \pm 0.4, p<0.001)$.

Antispasmodics and antidepressants improve IBS-D symptoms in $37 \%$ and $53 \%$ of patients respectively compared to the $71 \%$ of patients in this study implying that a low FODMAP diet is not only a safer treatment but also a more effective treatment ${ }^{(4)}$. However, due to the $50 \%$ response rate responder bias might have been present. The low diet is most effective at reducing abdominal discomfort it also significantly reduces loose bowel motions and urgency in IBS-D. The low FODMAP diet has been translated to clinical practice in the Northern region of England.

1. Maxion-Bergemann S, Thielecke F, Abel F et al. (2006) Costs of irritable bowel syndrome in the UK and US. Pharmacoeconomics. 24(1), 21-37.

2. Gibson PR \& Shepherd SJ (2010) Evidence-based dietary management of functional gastrointestinal symptoms: FODMAP approach. Journal of Gastroenterology and Hepatology 252-258.

3. Roalfe A, Roberts L, Wilson S Birmingham IBS Symptom Questionnaire. [Online]. Available from: http://www.biomedcentral.com/content/ supplementary/1471-230x-8-30-s2.pdf.

4. Ruepert L, Quartero A, de Wit N, et al. (2011) Bulking agents, antispasmodics and antidepressants for the treatment of irritable bowel syndrome. Cochrane Database Systematic Review. August 10; 8: p. CD003460. 